\title{
Welcher psychotherapeutische Zugang für den Grundversorger?
}

Korrespondenz:

Dr. med. Lucien Gamba

Facharzt Innere Medizin und Allergologie FMH, Bio-psycho-soziale Medizin (AMPP), Hypnose (SMSH) und Entspannungstherapie SBD (AMPP), Schweizerischer Verband der Psycho-Korporellen Therapien Boulevard Carl-Vogt 47

CH-1205 Genf
Lucien Gamba

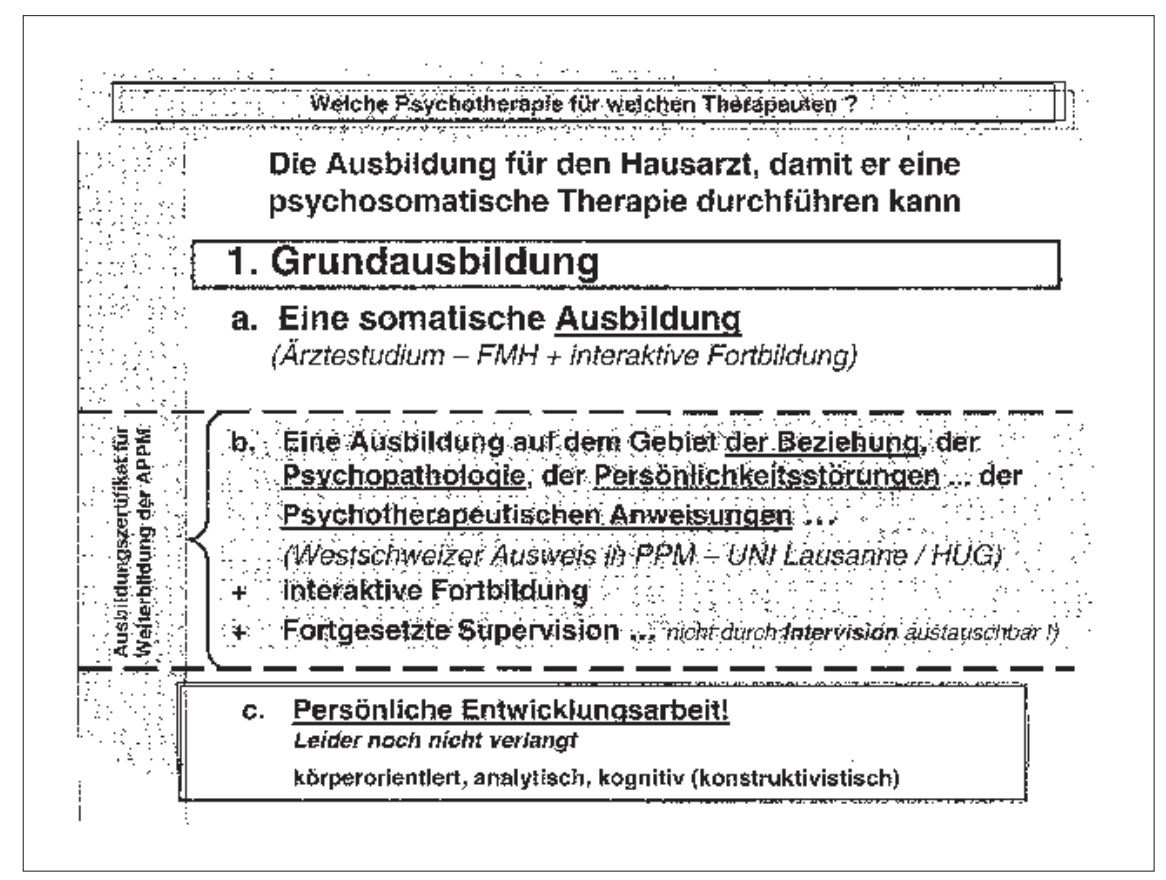

Zwar wird der psychosomatischen Dimension heutzutage im Rahmen der Postgraduate-Ausbildung etwas mehr Gewicht beigemessen als früher. Dennoch ist man noch weit von einem Standard entfernt, der die jungen Ärzte befähigt, den psychosomatischen Aspekt in ihr medizinisches Handeln zu integrieren. Dieses Manko ist so offensichtlich, dass die Grundversorger - sensibilisiert für diese umfassende Dimension der Betreuung des Patienten - eine ergänzende Ausbildung (APPM - Akademie für Psychosomatische und Psychosoziale Medizin) geschaffen haben. Diese Zusatzausbildung soll die mangelnde Kompetenz vermitteln, denn wenn Medizinalpersonen nicht die Fähigkeit besitzen, ein Vertrauensverhältnis aufzubauen, setzen sie kompensatorisch vermehrt medizinische Technik ein, und dies führt zu einer entsprechenden Erhöhung der Gesundheitskosten.

Solange im Medizinstudium nicht in jede therapeutische Handlung die Wiederherstellung des psychosomatischen Gleichgewichts integriert wird, ist es erforderlich, die umfassende Behandlung des Grundversorgers durch eine entsprechende, ergänzende Ausbildung zu vervollstän- digen. Diese soll den Mangel der «Pregraduate»Ausbildung kompensieren, indem sie sowohl eine theoretische Ausbildung als auch das tatsächliche Erleben vermittelt: Therapeutische Beziehung (Rollenspiel, Videosupervision), allgemeine Psychopathologie, Störungen der Persönlichkeit und verschiedene psychotherapeutische Indikationen. Diese Grundbildung muss durch eine Weiterbildung ergänzt werden sowie durch eine Supervision über einen längeren Zeitraum (und nicht nur durch Intervisionen, die sich als ungenügend erwiesen haben).

Infolge ihrer Spezifizität sollte die Fortbildung der Grundversorger in psychosomatischer Medizin genügend Raum in der Fortbildung einnehmen, das heisst, sie sollte mindestens 35\% (sogar bis $50 \%$ ) der jährlich geforderten 80 Stunden ausmachen. Nebenbei bemerkt sollten unter Berücksichtigung des Konzepts der «good practice» des Krankenversicherungsgesetzes diejenigen Hausärzte, die sich diese Ausbildung nicht angeeignet haben, in der Anzahl längerer psychotherapeutisch-psychosomatischer Gespräche pro Patient und pro Jahr begrenzt werden.

Es bleibt ein letztes wichtiges Problem: der psychosomatische Therapeut interessiert sich für den Patienten in seiner Gesamtheit und sucht die verborgenen Anteile seines Wesens auftauchen zu lassen. Dieser Behandlungsansatz kann nur durch ein Öffnen des emotionellen Raums des Patienten verwirklicht werden. Vom dem Moment an, da die therapeutische Beziehung eine Passung zwischen zwei Individuen darstellt, gilt folgendes: Wenn der Therapeut nicht in seinem eigenen emotionellen Raum an sich selbst arbeitet (die Wahl der Technik ist dabei nebensächlich), ist es illusorisch zu hoffen, dass sein Patient dies für sich tun wird. Eine Arbeit an der persönlichen Entwicklung müsste von den Grundversorgern gefordert werden, wenn er wünscht, längere psychotherapeutisch-psychosomatische Gespräche mit seinen Patienten zu führen.

Der Vorschlag ist, die psychosomatische Therapie als solche auch so zu benennen (um sie von der Psychotherapie des Psychiaters zu unterscheiden); die Handlung des Grundversorgers, der für die psychisch-physische Integration 

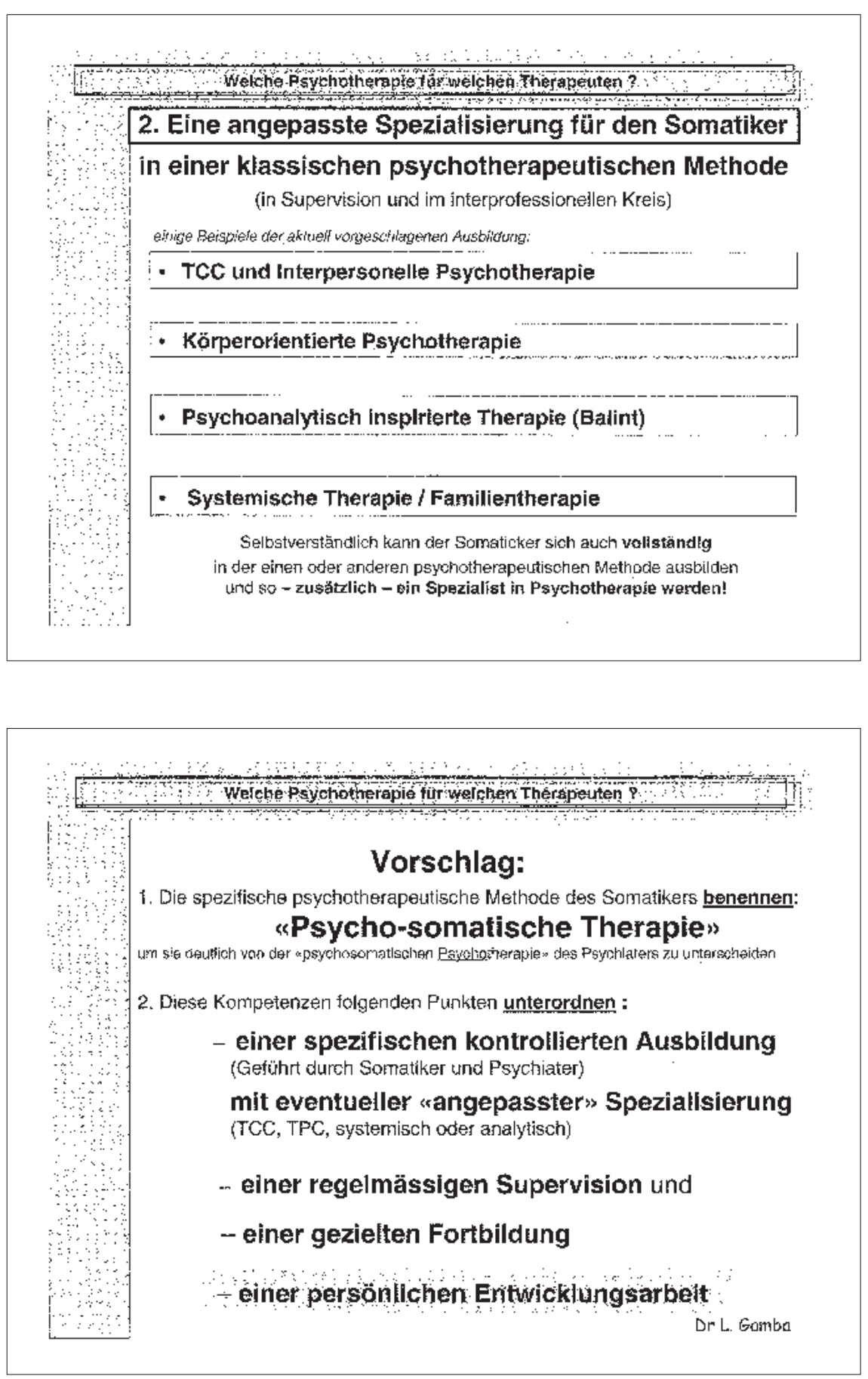

seiner Behandlungen ausgebildet ist, benötigt oft ein langsames Gewöhnen des Patienten an seine emotionelle Öffnung. Diese Öffnung braucht gewisse Techniken, die zur klassischen Psychotherapie gehören und die entsprechend an den psychosomatischen Raum des Hausarztes angepasst werden müssen, die aber sehr nützlich sind, um sich dem weniger bewussten Niveau des Symptoms anzunähern.

Die kognitive Verhaltenstherapie, die psychosomatische, die systemische und die analytische Therapie (letztere aber in geringerem Ausmass) bieten schon in den gemeinsam durch somatische Ärzte und Psychiater etablierten Programmen entsprechende Instrumente für den Gebrauch durch die Grundversorger. Diese sind nicht Psychotherapien im eigentlichen Sinn, aber ihr Einsatz (eine Verlängerung, da sie im «Sein in der therapeutischen Beziehung» agieren) führt zu Verbesserungen, auf die die Medizin nicht verzichten kann.

Das Symptom, ist es nicht letztlich eine Art von unbewusstem Vorwand, mit dem der Patient seinen Therapeuten auffordert, eventuell eines Tages «der Tutor seiner (des Symptoms) Auflösung» zu werden? 